\title{
Automatic ECG-based Discrimination of 20 Atrial Flutter Mechanisms: Influence of Atrial and Torso Geometries
}

\author{
Giorgio Luongo ${ }^{1}$, Steffen Schuler ${ }^{1}$, Massimo W Rivolta ${ }^{2}$, Olaf Dössel ${ }^{1}$, Roberto Sassi ${ }^{2}$, Axel Loewe ${ }^{1}$ \\ ${ }^{1}$ Institute of Biomedical Engineering, Karlsruhe Institute of Technology (KIT), Karlsruhe, Germany \\ ${ }^{2}$ Dipartimento di Informatica, Università degli Studi di Milano, Milan, Italy
}

\begin{abstract}
Atrial flutter (AFl) is a common heart rhythm disorder driven by different self-sustaining electrophysiological atrial mechanisms. In the present work, we sought to discriminate which mechanism is sustaining the arrhythmia in an individual patient using non-invasive 12-lead electrocardiogram (ECG) signals. Specifically, we analyse the influence of atrial and torso geometries for the success of such discrimination. 2,512 ECG were simulated and 151 features were extracted from the signals. Three classification scenarios were investigated: random set classification; leave-one-atrium-out (LOAO); and leave-onetorso-out (LOTO). A radial basis neural network classifier achieved test accuracies of $89.84 \%, 88.98 \%$, and $59.82 \%$ for the random set classification, LOTO, and LOAO, respectively. The most discriminative single feature was the $F$-wave duration (74\% test accuracy). Our results show that a machine learning approach can potentially identify a high number of different AFl mechanisms using the 12lead ECG. More than the 8 atrial models used in this work should be included during training due to the significant influence that the atrial geometry has on the ECG signals and thus on the resulting classification. This non-invasive classification can help to identify the optimal ablation strategy, reducing time and resources required to conduct invasive cardiac mapping and ablation procedures.
\end{abstract}

\section{Introduction}

Atrial flutter (AFl) is an atrial tachycardia characterized by electrical signals that repeatedly propagate along various physiological pathways different from sinus rhythm [1]. This arrhythmia is driven by different selfsustaining reentrant mechanisms. Although AFl is not a direct cause of death, it can cause significant symptoms and complications - e.g., stroke and heart attacks. The most commonly used treatment to restore sinus rhythm from AFl conditions is ablation therapy. The problem of ablation therapy consists in identifying the type of $\mathrm{AFl}$, as each mechanism requires a different ablation procedure. Therefore, the doctor needs to know clearly the type of AFl in progress before performing the ablation. To identify the AFl mechanism, an invasive mapping of the electrical activity of the atria is carried out using intracardiac catheters.

Using the 12-lead ECG to discriminate the type of AFl with which the patient is affected would give doctors the opportunity to plan the intervention in advance. Thus, reducing the procedure time for invasive mapping and ablation therapy. Multiple algorithms have been proposed to discriminate $\mathrm{AFl}$ from other types of cardiac arrhythmias, such as atrial fibrillation [2,3]. Nevertheless, automatic discrimination of different AFl mechanisms has not yet been carried out.

In this preliminary study, we sought to discriminate 20 different simulated AFl mechanisms by using 12-lead ECG signals. Moreover, we focused on the role of the geometries of the atria and torso for the success of this automatic discrimination.

\section{Methods}

\subsection{Simulated AFI scenarios}

Based on the AFl mechanisms commonly differentiated in literature $[4,5]$, precisely resembling documented clinical AFl cases [6-8], and the simulations implemented in a previous work by Oesterlein et al. [9], a database of computational AFl scenarios was set up.

Cardiac excitation was modelled using the fast marching approach to solve the Eikonal equation [10,11].

The atrial electrophysiological activity was simulated on the tetrahedral volume meshes of 8 bi-atrial anatomies, generated from segmented magnetic resonance imaging (MRI) data of healthy male and female subjects [12].

In total, 20 mechanisms/scenarios of AFl were implemented, including right atrial (RA) flutter as well as left atrial (LA) forms like macroreentry around the valves (sc14 ), across the roof (sc9-11), focal ectopy (sc12-15), and microreentries mediated by scars (sc5-8), and slow conduction areas (sc16-20). A complete list of scenarios is 
Table 1. Database of clinically informed manually parameterized AFl mechanisms

\begin{tabular}{|c|c|c|c|c|}
\hline Mechanism & Atrium & Position & Direction & ID \\
\hline Macroreentry & RA & Tricuspid Valve & $\mathrm{ccw}$ & $\mathrm{sc} 1$ \\
\hline Macroreentry & RA & Tricuspid Valve & $\mathrm{cw}$ & $\mathrm{sc} 2$ \\
\hline Macroreentry & LA & Mitral Valve & $\mathrm{ccw}$ & $\operatorname{sc} 3$ \\
\hline Macroreentry & LA & Mitral Valve & $\mathrm{cw}$ & $\operatorname{sc} 4$ \\
\hline Scar-related Reentry & LA & LPV & post & $\operatorname{sc} 5$ \\
\hline Scar-related Reentry & LA & LPV & ant & sc6 \\
\hline Scar-related Reentry & LA & RPV & post & sc7 \\
\hline Scar-related Reentry & LA & RPV & ant & $\operatorname{sc} 8$ \\
\hline Figure-8 Macroreentry & LA & Both PVs & ant & sc9 \\
\hline Figure-8 Macroreentry & LA & Both PVs & post & $\mathrm{sc} 10$ \\
\hline Figure-8 Macroreentry & LA & RPVs & ant & $\mathrm{sc} 11$ \\
\hline Focal Source & LA & RSPV anterior & & $\mathrm{sc} 12$ \\
\hline Focal Source & LA & RSPV posterior & & $\mathrm{sc} 13$ \\
\hline Focal Source & LA & LSPV anterior & & $\mathrm{sc} 14$ \\
\hline Focal Source & LA & LSPV posterior & & $\mathrm{sc} 15$ \\
\hline Microreentry & LA & ant MV annulus & & $\mathrm{sc} 16$ \\
\hline Microreentry & LA & ant LAA & & $\mathrm{sc} 17$ \\
\hline Microreentry & LA & ant RSPV & & $\mathrm{sc} 18$ \\
\hline Figure- 8 Microreentry & LA & ant & & $\operatorname{sc} 19$ \\
\hline Microreentry & LA & post wall & & $\operatorname{sc} 20$ \\
\hline
\end{tabular}

$\overline{\overline{\text { Right atrium (RA), left atrium (LA), left pulmonary vein (LPV), right }}}$ pulmonary vein (RPV), pulmonary veins (PVs), right superior pulmonary vein (RSPV), left superior pulmonary vein (LSPV), mitral valve (MV), left atrial appendage (LAA), clockwise (cw), counterclockwise (ccw), anterior (ant), posterior (post), scenario (sc).

provided in Table 1.

Transmembrane voltages (TMV) were obtained by aligning a template of their time course with activation times. This TMV was calculated using the Courtemanche et al.'s mathematical model of the human atrial action potential including chronic atrial fibrillation remodeling [13]. From the TMV, the body surface potential map (BSPM) was calculated on 8 different triangulated torso surface models generated from segmented MRI data of healthy male and female subjects $[12,14]$. The boundary element method was used to solve the forward problem of electrophysiology [15]. From the BSPM, the 12-lead ECG was extracted, including the 12-lead ECGs. A detailed description of the simulation procedure can be found in [16].

Each 12-lead ECG signal has a length of a single AFl loop and a sample frequency of $1 \mathrm{kHz}$. The 12-lead ECG signals are formed only by F-waves (flutter waves, Pwaves during $\mathrm{AFl}$ ) without the QRS complex and T-wave (representing ventricular activity) since the ventricles were not included in the simulations (Fig. 1A-C).

A total of 2,512 12-lead ECGs were calculated from 20 simulated AFl scenarios on 8 atrial models with two orientational variants each and 8 torso models. One of the atrial models was not able to sustain sc12, sc13, and sc30 for geometric reasons. Therefore, these AFl scenarios were not computed with this geometry.

\subsection{Correlation analysis}

The influence of atrial and torso geometry was assessed by circular cross-correlation analysis. Correlation analysis was performed between ECG signals with different atrial models, keeping AFl mechanisms and torso models fixed. The same procedure was applied between ECG signals with different torso models while keeping AFl mechanisms and atrial models fixed. The correlation coefficients obtained were merged by averaging along the 12 leads.

\subsection{Feature extraction}

151 features were extracted from the 12-lead ECGs using several biosignal processing methods from different domains, i.e., time, frequency, wavelet, entropy, and nonlinear recurrence analysis.

Among these features, and looking at the results, the Fwave duration feature proved to be particularly relevant. The F-wave duration was the duration time of a complete cycle of atrial electrical activation of each specific AFl mechanism, i.e., the length of the F-wave in the ECG signal. This feature was manually derived.

\subsection{Feature selection}

Greedy forward selection algorithm was implemented to select a feature set. This algorithm started with an empty feature set and added the feature leading to the highest accuracy increase to the set at each iteration. The algorithm was stopped when performance based on the validation set could not be further increased. In order to handle possible correlations among features, the candidate feature to be added to the set was only added if the correlation coefficient with any of the already included features was $<0.6$.

\subsection{Classification}

Three classifiers were implemented for a 20 classes discrimination: decision tree (DT), k-nearest neighbours $(\mathrm{KNN})$, and radial basis neural network ( $\mathrm{rbNN})$.

First, we wanted to simulate the scenario of having similar geometries in each data subset. Therefore, the data were randomly divided in training set, validation set, and test set with ratios of $70 \%, 15 \%$, and $15 \%$, respectively. Second, classifications were performed with leave-oneatrium-out (LOAO) and leave-one-torso-out torso (LOTO) algorithms. In the LOAO algorithm, 7 atrial geometries were used in the training set and the remaining atrial geometry was used both in the validation and test set (50\% of the ECGs from this geometry in each set). This procedure was cyclically repeated 8 times always changing the validation/test atrial geometry. The average accuracy of 
A. Simulated atrial flutter

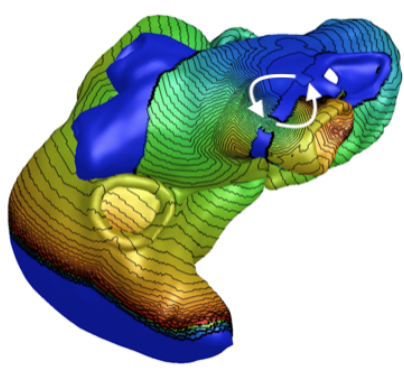

B. Body surface potential map

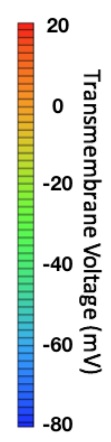

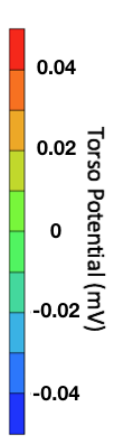

c.

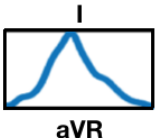

aVR

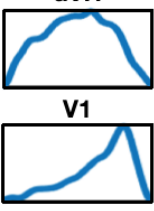

V4

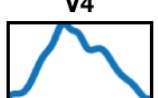

ECG

$\mathrm{mv}$
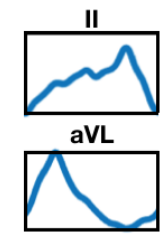

V2

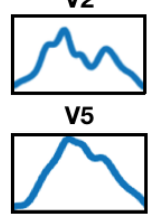

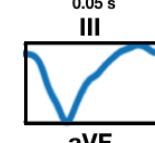
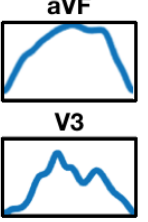

V6

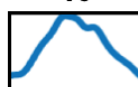

Figure 1. A. Simulated scar-related reentry AFl located on the LPV with anterior direction of rotation (sc6 - white arrows) on an atrial model generated from MRI. B. BSPM on torso model generated from MRI. The torso potential was obtained by solving the forward problem of electrophysiology from the simulated TMV on the atria. C. Example of the F-wave single loop of the 12-lead ECG signals extracted from the BSPM.

the 8 loops was used as performance parameter. The same procedure was applied for the LOTO.

\section{Results}

\subsection{Torsos and atrial models influence on the ECG}

The correlation distribution obtained from the correlation analysis between ECG signals of the same AFl types computed in the same torsos but on different atria showed a median of 0.44 and an interquartile range (IQR) of 0.13. On the contrary, a median of 0.78 and IQR of 0.11 was found from the correlation analysis between ECG signals of the same AFl types with the same atria but different torsos. Fig. 2 shows an example of the effect that different torso geometries and atrial geometries have on the 12-lead ECGs (Fig. 2A. and B. respectively).

\subsection{Random set classification}

The rbNN achieved the highest performance with $89.84 \%$ accuracy on the test set using 10 features. The KNN and DT classifiers achieved $83.25 \%$ and $81.02 \%$ accuracy on the test set using 12 and 5 features, respectively.

F-wave duration was the most discriminative feature for all classification algorithms. This single feature classified the AFl mechanisms with a test accuracy of $74 \%$, while the entire feature set without F-wave duration reduced the test set accuracy to $33 \%$ (rbNN).
A.

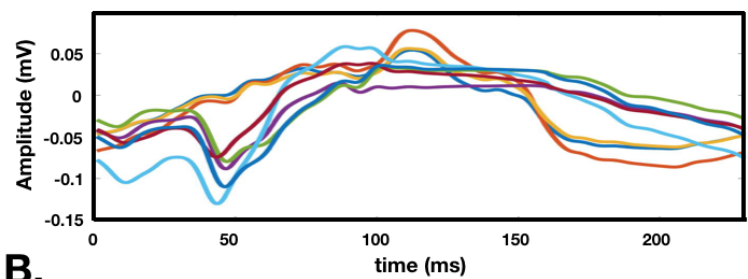

B.

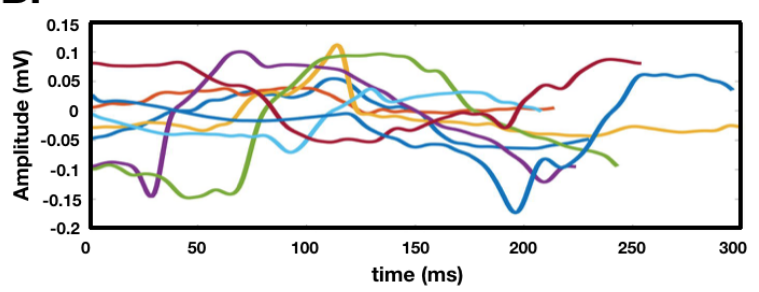

Figure 2. Example of simulated ECG (lead I) of the AFl scenario $s c l$ with different torso and atrial models. A. Lead I simulated on the same atria model with 8 different torso geometries. B. Lead I simulated on 8 different atria models with the same torso geometry.

\subsection{LOTO \& LOAO}

The LOTO and LOAO classifications with rbNN yielded a test accuracy of $88.98 \%$ and $59.82 \%$ using 7 and 6 features, respectively. In both cases, the F-wave duration was selected as the first feature in the feature set.

\section{Discussion and Conclusions}

Simulations provide ideal and controlled scenarios where the ground truth for $\mathrm{AFl}$ perpetuation is known in all cases, allowing the analysis of each mechanism with- 
out the influence of secondary - or unknown - mechanisms, e.g., other simultaneous AFls.

The results obtained with the random set classification show that an automatic classifier can potentially identify a high number of different AFl mechanisms using the 12lead ECG, or more precisely a single F-wave loop, when similar geometries are present in each data subset. This non-invasive method can help physicians to plan the most appropriate treatment for the patients without the need of prior invasive mapping.

The F-wave duration is a key feature for this classification. The LOTO accuracy shows that the classifier is generalizing well to unseen torso geometries. The LOAO accuracy shows a lack of ability to generalize with new atrial geometries. These last three considerations can be linked since atrial geometries have an influence on the F-wave duration and on the ECG signals (Fig. 2B.). On the contrary, an additional torso to the set of 7 used for training does not yield much benefit, because different torso geometries do not bring relevant changes on the F-wave duration and on the ECG signals in general (Fig. 2A.), as also confirmed by the correlation analysis. Therefore, more than the currently used 8 atrial models should be included during training to cover the relevant anatomical variability.

Further tests on clinical data are necessary to effectively assess the proposed approach. Changes in conduction velocity would also change the F-wave duration irrespective of the geometry and should therefore also be varied.

\section{Acknowledgments}

Research supported by H2020-MSC No.766082 (MYATRIA). All authors confirm that they have no other relationships relevant to the contents of this paper to disclose.

\section{References}

[1] Cosio FG, Pastora A, Núñeza A, Magalhaesa AP, Awamleh P. Atrial flutter: an update. Rev Esp Cardiol 2006; 59(8):816-831.

[2] Acharya UR, Fujita H, Adam M, Lih OS, Hong TJ, Sudarsha VK, Koh JE. Automated characterization of arrhythmias using nonlinear features from tachycardia ecg beats. IEEE SMC Conference 2016;533-538.

[3] Taha B, Reddy S, Xue Q, Swiryn S. Automated discrimination between atrial fibrillation and atrial flutter in the resting 12-lead electrocardiogram. J Electrocardio 2000;33:123125.

[4] Jais J, Matsuo S, Knecht S, Weerasooriya R, Hocini M, Sacher F, Wright M, Nault I, Lellouche N, Klein G, Clémenty J, Haïssaguerre M. A deductive mapping strategy for atrial tachycardia following atrial fibrillation ablation: importance of localized reentry. J Cardiovasc Electrophysiol 2009;20(5):480-491.

[5] Ban JE, Chen YL, Park HC, Lee HS, Lee DI, Choi JI,
Lim HE, Park SW, Kim YH. Relationship between complex fractionated atrial electrograms during atrial fibrillation and the critical site of atrial tachycardia that develops after catheter ablation for atrial fibrillation. J Cardiovasc Electrophysiol 2014;25(2):146-153.

[6] Schilling RJ, Peters NS, Goldberger J, Kadish AH, Davies DW. Characterization of the anatomy and conduction velocities of the human right atrial flutter circuit determined by noncontact mapping. J Am Coll Cardiol 2001;38(2):385393.

[7] Ndrepepa G, Zrenner B, Weyerbrock S, Schneider MA, Schmitt C. Activation patterns in the left atrium during counterclockwise and clockwise atrial flutter. J Cardiovasc Electrophysiol 2001;12(8):893-899.

[8] Markowitz SM, Thomas G, Liu CF, Cheung JW, Ip JE, Lerman BB. Atrial tachycardias and atypical atrial flutters: mechanisms and approaches to ablation. Circ Arrhythm Electrophysiol 2019;8(2):131-137.

[9] Oesterlein TG, Loewe A, Lenis G, Luik A, Schmitt C, Dössel O. Automatic identification of reentry mechanisms and critical sites during atrial tachycardia by analyzing areas of activity. IEEE Trans Biomed Eng 2018;65(10):23342344.

[10] Jacquemet V. An eikonal approach for the initiation of reentrant cardiac propagation in reaction-diffusion models. IEEE Trans Biomed Eng 2010;57(9):2090-2098.

[11] Trächtler J, Oesterlein T, Loewe A, Poremba E, Luik A, Schmitt C, Dössel O. Virtualizing clinical cases of atrial flutter in a fast marching simulation including conduction velocity and ablation scars. CDBME 2015;1(1):405-408.

[12] Krueger MW, Seemann G, Rhode K, Keller DU, Schilling C, Arujuna A, Gill J, O’Neill MD, Razavi R, Dössel O. Personalization of atrial anatomy and electrophysiology as a basis for clinical modeling of radio-frequency ablation of atrial fibrillation. IEEE Trans Med Imaging 2013;32(1):7384.

[13] Wilhelms ALM, Dössel O, Seemann G. Influence of chronic atrial fibrillation induced remodeling in a computational electrophysiological model. Biomedizinische Technik 2014;59(S1):S929-S932.

[14] Lux RL. Body surface potential mapping techniques. Comprehensive Electrocardiology 2010;1361-1374.

[15] Stenroos M. The transfer matrix for epicardial potential in a piece-wise homogeneous thorax model: The boundary element formulation. Phys Med Biol 2009;54(18):54435455.

[16] Luongo G, Schuler S, Luik A, Almeida TP, Soriano DC, Dössel O, Loewe A. Non-invasive characterization of atrial flutter mechanisms using recurrence quantification analysis on the ecg: a computational study. IEEE Trans Biomed Eng 2020; accepted.

Address for correspondence:

Giorgio Luongo, Karlsruhe Institute of Technology (KIT)

Fritz-Haber-Weg 1, 76131 Karlsruhe, Germany

publications@ibt.kit.edu 